\title{
Mixed rain forest in southeastern Brazil: tree species regeneration and floristic relationships in a remaining stretch of forest near the city of Itaberá, Brazil
}

Tiago Maciel Ribeiro ${ }^{1}$, Natália Macedo Ivanauskas²,5, Sebastião Venâncio Martins ${ }^{1}$, Rodrigo Trassi Polisel ${ }^{3}$, Rochelle Lima Ramos dos Santos ${ }^{4}$ and Aurino Miranda Neto ${ }^{1}$

Submitted: 14 December, 2011. Accepted: 25 October, 2012

\begin{abstract}
The aim of this work was to evaluate the floristic composition, richness, and diversity of the upper and lower strata of a stretch of mixed rain forest near the city of Itaberá, in southeastern Brazil. We also investigated the differences between this conservation area and other stretches of mixed rain forest in southern and southeastern Brazil, as well as other nearby forest formations, in terms of their floristic relationships. For our survey of the upper stratum (diameter at breast height $[\mathrm{DBH}] \geq 15 \mathrm{~cm}$ ), we established 50 permanent plots of $10 \times 20 \mathrm{~m}$. Within each of those plots, we designated five, randomly located, $1 \times 1 \mathrm{~m}$ subplots, in order to survey the lower stratum (total height $\geq 30 \mathrm{~cm}$ and $\mathrm{DBH}<15 \mathrm{~cm}$ ). In the upper stratum, we sampled 1429 trees and shrubs, belonging to 134 species, 93 genera, and 47 families. In the lower stratum, we sampled 758 trees and shrubs, belonging to 93 species, 66 genera, and 39 families. In our floristic and phytosociological surveys, we recorded 177 species, belonging to 106 genera and 52 families. The Shannon Diversity Index was 4.12 and 3.5 for the upper and lower strata, respectively. Cluster analysis indicated that nearby forest formations had the strongest floristic influence on the study area, which was therefore distinct from other mixed rain forests in southern Brazil and in the Serra da Mantiqueira mountain range.
\end{abstract}

Key words: Araucaria angustifolia, Cluster analysis, ecotone, phytogeography, phytosociology

\section{Introduction}

When attributed to mixed rain forest, the term "mixed" refers to the presence of the gymnosperms Araucaria angustifolia (Bertol.) Kuntze (Brazilian pine) and Podocarpus lambertii Klotzsch ex Endl. (pinheiro-bravo) in association with primitive angiosperm genera, such as Drimys (Winteraceae), as well as Ocotea, Cryptocarya and Nectandra (Lauraceae). Klein (1960) divides Araucaria ecosystems into various successional stages, attributing the term "mixed forest" to their more mature stage. Veloso (1991) attributes the term "mixed rain forest" to all ecosystems containing A. angustifolia, which is always associated with broadleaf species. Rizzini (1997) supports the classification made by Klein (1960), stating that Araucaria forests represent the intermediate successional stage of mature forests without Araucaria. Therefore, there is no consensus on the classification of Araucaria forests. According to Jarenkow \& Budke (2009), although the physiognomy might suggest some uniformity due to the occurrence of emergent Araucaria, floristic and phytosociological inventories reveal particular distinctions throughout the various Araucaria forests.

A large part of the remaining fragments of mixed rain forest in the state of São Paulo is located in the southern region of the state, near the state of Paraná, in the Upper Paranapanema River watershed. These fragments collectively cover an estimated 131 ha, only $14 \%$ of which corresponds to primary forest. The remainder consists of forest refuges at high altitudes in the Serra do Mar and Mantiqueira mountain ranges, accounting for 42,805 ha, only $39 \%$ of which is primary forest (Klein 1960; Veloso et al. 1991).

The current critical state of conservation of the mixed rain forest in Brazil calls for the collection of basic information about the composition, structure, richness and successional dynamics of its physiognomy, as well as the expansion of knowledge regarding the floristic and structural variations among the various stretches within its natural range, in order to inform decision-making regarding the conservation and

\footnotetext{
${ }^{1}$ Universidade Federal de Viçosa, Departamento de Engenharia Florestal, Viçosa, MG, Brazil

${ }^{2}$ Instituto Florestal, Seção de Ecologia Florestal, São Paulo, SP, Brazil

${ }^{3}$ Universidade Estadual de Campinas, Instituto de Biologia, Campinas, SP, Brazil

${ }^{4}$ Faculdade São Judas Tadeu, São Paulo, SP, Brazil.

${ }^{5}$ Author for correspondence: nivanaus@yahoo.com.br
} 
restoration of these ecosystems, thus increasing the chances of success in the long term. However, such aspects merit in-depth scientific investigation, especially for stretches of forest in the state of São Paulo.

The purpose of this study was to characterize the floristic composition, richness and diversity of the upper and lower strata of a stretch of Araucaria forest in the state of São Paulo, investigating the floristic similarity between them. In addition, we sought to arrive at a more realistic classification of the vegetation in the area, taking into account the floristic composition of the current upper stratum and possible trajectories revealed by the lower stratum, not just its physiognomic character (mainly of the canopy). In short, we attempted to assess whether the fragment under study exhibited sufficient species richness (of trees and shrubs) to classify it as a typical remnant of mixed rain forest, investigating its floristic relationship with stretches of the same formation in southern Brazil and within the same state (São Paulo), in addition to surveys in the dense rain forest, semideciduous seasonal forest and deciduous forest within the state.

\section{Materials and methods}

\section{Study area}

The study was conducted at the Itaberá Ecological Station (located in the municipality of Itaberá, in the state of São Paulo), which is operated by the São Paulo State Forestry Institute and was created by decree (State Decree no. 26.890/1987). The area is located in the watershed of the Upper Paranapanema River, in the southern region of the state ( $\left.23^{\circ} 50^{\prime} 47^{\prime \prime} \mathrm{S} ; 49^{\circ} 08^{\prime} 39^{\prime \prime} \mathrm{W}\right)$. The Station preserves a fragment of mixed rain forest of approximately 180 ha drained by three tributaries of the Ribeirão das Lavrinhas River, at altitudes ranging from $680 \mathrm{~m}$ to $710 \mathrm{~m}$.

The climate is temperate, without a true dry season ( $\mathrm{Cfb}$ in the Köppen climate classification system). The hottest month is February, during which the average temperature is $22.5^{\circ} \mathrm{C}$, and the lowest temperatures are observed between May and August, the monthly averages then ranging from $15.0^{\circ} \mathrm{C}$ to $16.7^{\circ} \mathrm{C}$ (observations from 1979 to 1990 ; Sentelhas et al. 1999). The average annual rainfall is $1405 \mathrm{~mm}$, with monthly rainfall averages ranging from $47 \mathrm{~mm}$ in July to $184 \mathrm{~mm}$ in January, according to data from the Apiaí weather station, which is the weather station closest to the study area (Souza 2008).

In the interfluves of the area, there is red latosol (oxisol), red nitosol in the steeper areas and gleysol at the valley floors (Novais et al. 2009). The vegetation matrix of the region is composed of agricultural crops on gently curving terraces, which allows the mechanization of the various stages of production, interspersed with rangeland, forest plantations and small isolated fragments of forest. In the forest inventory of the state of São Paulo (Kronka et al.2005), the vegetation of the area was officially classified as mixed rain forest.

\section{Sampling and data collection}

For the phytosociological inventory, we selected an area that was relatively uniform in terms of topography and soil conditions (interfluves with gentle slopes, on red latosol), representative of the predominant condition of the Station. The area selected was in the interior of the fragment under study and thus free from the interference of the edge effect.

For our survey of the upper stratum, we established 50 permanent plots of $10 \times 20 \mathrm{~m}$, arranged contiguously and totaling 1 ha. We defined the upper stratum as trees and shrubs with a diameter at breast height (DBH, $1.3 \mathrm{~m})$ $\geq 15 \mathrm{~cm}$. For individuals ranging below breast height, we included only those that met the inclusion criterion for at least one of their branches.

To survey the lower stratum, we established, within each $10 \times 20 \mathrm{~m}$ plot, five $1 \times 1 \mathrm{~m}$ subplots, totaling $250 \mathrm{~m}^{2}$ of the sample area (Fig. 1). The positioning of subplots was drawn by lot of coordinates of the upper left vertex, taking as reference the sides of the $10 \times 20 \mathrm{~m}$ plot, known as Cartesian axes. To measure these coordinates in the field, we used a vertex. We sampled all tree or shrub individuals that were $\geq 30 \mathrm{~cm}$ in height and had a DBH $<15 \mathrm{~cm}$.

Most of the individuals sampled in the upper and lower strata were identified in the field. When that was not possible, we collected botanical material and recorded dendrological characteristics (bark, flower/fruit color, scent, presence of exudates, etc.) for later identification. Aiming to expand the floristic survey, we also walked cross-country and drove motor vehicles along the principal trails of the Station to collect reproductive material of species that were or were not present in the phytosociological inventory plots.

All botanical material was duly numbered, arranged in presses, dehydrated and delivered to the Forest Ecology Sector of the São Paulo Forestry Institute, where it was identified through comparisons with materials at the Dom Bento Pickel Herbarium (code, SPSF), as well as by consulting experts of recognized competence and the specialized literature. The fertile specimens were incorporated into the collection of the same herbarium. We adopted the classification system proposed by the Angiosperm Phylogeny Group (APG III, 2009).

In order to assess the statistical power of the sampling used in each component, we plotted species accumulation curves by re-sampling individuals, with 10,000 interactions and $95 \%$ confidence intervals (Gotelli \& Colwell 2001).

\section{Statistical analysis}

For both strata, we estimated the Shannon diversity index (H', using a neperian logarithm and 95\% jackknife confidence intervals), Pielou's evenness index (J'; Pielou 1966) and the Jentsch mixture coefficient (MC; Hosokawa 1981). For this analysis, we used the Mata Nativa software (Cientec 2004). 


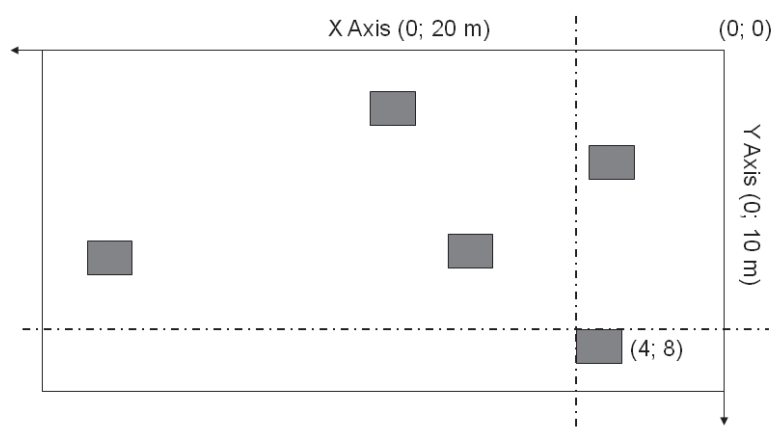

个 N $\square 10 \times 20 \mathrm{~m}$ plot $\square 1 \times 1 \mathrm{~m}$ subplot

Figure 1. Sketch showing the location of five $1 \times 1 \mathrm{~m}$ subplots within a $10 \times 20$ $\mathrm{m}$ plot (multi-level sampling), in a fragment of mixed rain forest at the Itaberá Ecological Station, Itaberá, in the state of Sãoa Paulo, Brazil. The dashed line shows the position of a subplot, selected by random drawing of coordinates of the upper left vertex, the reference points being the sides of the $10 \times 20 \mathrm{~m}$ plot, which were taken as Cartesian axes.

We analyzed the floristic similarity between the upper and lower strata in order to detect possible changes between the current floristic composition, revealed by species present in the upper stratum, and possible trajectories associated with natural regeneration dynamics in the study area, inferred from the lower stratum. In this analysis, we used the Sorensen and Jaccard similarity indices.

In order to investigate the floristic relationship between the Itaberá Ecological Station and other stretches of mixed rain forest in southern and southeastern Brazil, as well as other formations surrounding the Station, we selected 52 phytosociological surveys conducted in the three southern states other than São Paulo, in upper montane, montane and submontane environments (Table 1). We gave preference to surveys with a minimum of one hectare of sampling area in which the inclusion criterion was $\mathrm{DBH} \geq 4.8 \mathrm{~cm}$. Nevertheless, due to the small number of and limited access to such studies, we considered some surveys that did not meet our requirements in terms of the sampling area and the inclusion criterion.

Based on floristic lists of each work, we prepared a binary presence/absence matrix, with species listed in rows and areas in columns. We included only the data related to specimens identified down to the species level, and we consulted the Botanical Garden of Rio de Janeiro (2010) to update the scientific names of species, including the occurrence of synonyms. The classification method used was hierarchical cluster analysis, which generates a dendrogram showing a hierarchical organization of the groups formed. As the coefficient, we used the Jaccard similarity index, with an agglomerative algorithm (the unweighted pair group method with arithmetic mean). In this analysis, we used the FITOPAC 1 software (Shepherd 1995).

To identify threatened species at the Itaberá Ecological Station, we consulted the Official List of Threatened Species in the State of São Paulo, issued by the São Paulo State Secretaria do Meio Ambiente (SMA, Department of the Environment; Resolution no. 48; São Paulo 2004), the Official List of Endangered Species in Brazil, issued by the Instituto Brasileiro do Meio Ambiente e dos Recursos Naturais Renováveis (IBAMA, Brazilian Institute for the Environment and Renewable Natural Resources; Directive no. 37; IBAMA 1993) and the International Union for Conservation of Nature (IUCN) Red List of Globally Threatened Species (IUCN 1994/2001). This analysis aimed to determine the relevance of this area of full protection of target plant populations, considering the critical state of conservation of the mixed rain forest in São Paulo.

\section{Results and Discussion}

\section{Floristic composition}

In the upper stratum, we sampled 1429 trees and shrubs belonging to 134 species, 93 genera and 47 families, one individual being identified only down to the genus level. In the lower stratum, we sampled 758 individuals belonging to 93 species, 66 genera and 39 families, one individual being identified only down to the family level only and another being identified only down to the genus level. In the upper and lower strata, we sampled, respectively, 75 and 4 standing dead individuals $(5.25 \%$ and $0.53 \%$ of the total). In the phytosociological survey as a whole, we identified 161 species belonging to 104 genera and 51 families.

As of the 46th plot and 224th subplot in the upper and lower strata, respectively, the collector curve showed some stabilization, indicating the statistical power of the sampling. However, through the floristic survey conducted on several Station trails, we collected 16 species not sampled in the phytosociological survey, belonging to 16 genera and 13 families. Therefore, the total number of species recorded at the Itaberá Ecological Station in the present study rose to 177, representing 106 genera and 52 families (Table 2). The species that were exclusive to our floristic survey accounted for $8.9 \%$ of the species observed, which demonstrates its additional contribution to the knowledge of the local flora. This information could inform decisions regarding future management plans, forest restoration projects in surrounding areas, and environmental education initiatives aimed at raising awareness among the population about the need to and importance of preserving these last living witnesses to the original landscape.

The most species-rich families in the upper stratum were Lauraceae and Myrtaceae (12 species each); Rubiaceae (8 species); Euphorbiaceae, Fabaceae-Faboideae, Meliaceae and Salicaceae (6 species each); and Fabaceae-Mimosoideae, Rutaceae and Sapindaceae (5 species each). In the lower stratum, these were Myrtaceae (10 species); Rubiaceae (8 species); Melastomataceae (6 species); Meliaceae (5 species); and Fabaceae-Faboideae, Lauraceae, Fabaceae-Mimosoideae, Sapindaceae and Solanaceae ( 4 species each). It is of note that there was an abundance of families represented by a 
Table 1. Phytosociological surveys used for floristic comparison among areas of mixed rain forest, dense rain forest, semideciduous seasonal forest and deciduous seasonal forest in the Brazilian states of São Paulo, Paraná, Santa Catarina and Rio Grande do Sul.

\begin{tabular}{|c|c|c|c|c|c|c|c|c|}
\hline LC & Reference & Municipality - state & $\mathrm{CF}$ & Lat & Long & Alt & DBH & SR \\
\hline AP & Godoy 2001 & Apiaí and Iporanga - SP & MD & - & - & 600 & 4.8 & 49 \\
\hline AP1 & Godoy 2001 & Apiaí and Iporanga - SP & MD & - & - & 600 & 4.8 & 44 \\
\hline AP2 & Godoy 2001 & Apiaí and Iporanga - SP & MD & - & - & 670 & 4.8 & 92 \\
\hline $\mathrm{BC}$ & Souza 2008 & Barra do Chapéu - SP & $\mathrm{MM}$ & $24^{\circ} 28^{\prime}$ & $49^{\circ} 01^{\prime}$ & 900 & 4.8 & 123 \\
\hline СВ & Custódio Filho 2002 & Capão Bonito - SP & MD & $24^{\circ} 00^{\prime}$ & $48^{\circ} 00^{\prime}$ & 600 & 10.0 & 88 \\
\hline CÇ & Negrelle et al. 1992 & Caçador - SC & $\mathrm{UM}$ & $26^{\circ} 47^{\prime}$ & $51^{\circ} 01^{\prime}$ & 1100 & 5.0 & 43 \\
\hline CJ1 & Los 2004 & Campos do Jordão - SP & $\mathrm{UM}$ & $22^{\circ} 45^{\prime}$ & $45^{\circ} 30^{\prime}$ & 1467 & 5.0 & 120 \\
\hline CJ2 & Souza 2008 & Campos do Jordão - SP & $\mathrm{UM}$ & $22^{\circ} 41^{\prime}$ & $45^{\circ} 27^{\prime}$ & 1500 & 4.8 & 58 \\
\hline $\mathrm{CM}$ & Cielo Filho \& Santin 2002 & Campinas - SP & MS & $22^{\circ} 53^{\prime}$ & $47^{\circ} 04^{\prime}$ & 685 & 4.8 & 105 \\
\hline $\mathrm{CR}$ & Rondon Neto et al. 2002(a) & Criúva - RS & MM & $29^{\circ} 00^{\prime}$ & $55^{\circ} 56^{\prime}$ & 860 & 5.0 & 37 \\
\hline CS & Formento et al. 2004 & Campo Belo do Sul - SC & $\mathrm{UM}$ & $28^{\circ} 00^{\prime}$ & $50^{\circ} 49^{\prime}$ & 1017 & 3.3 & 70 \\
\hline CT1 & Rondon Neto et al. 2002 (b) & Curitiba - PR & MM & $25^{\circ} 26^{\prime}$ & $49^{\circ} 14^{\prime}$ & - & 5.0 & 77 \\
\hline CT2 & Kozera et al. 2005(a) & Curitiba - PR & $\mathrm{MM}$ & - & - & 900 & 3.3 & 103 \\
\hline CT3 & Kozera et al. 2005(b) & Curitiba - PR & MM & - & - & 900 & 10.0 & 77 \\
\hline GA & Durigan et al. 2000 & Gália - SP & MS & $22^{\circ} 24^{\prime}$ & $49^{\circ} 42^{\prime}$ & 600 & 5.0 & 62 \\
\hline GC & Watzlawick et al. 2005 & General Carneiro - PR & MM & $26^{\circ} 26^{\prime}$ & $51^{\circ} 25^{\prime}$ & 900 & 3.3 & 39 \\
\hline GP1 & Cordeiro \& Rodrigues 2007 & Guarapuava -PR & $\mathrm{UM}$ & $25^{\circ} 21^{\prime}$ & $51^{\circ} 28^{\prime}$ & 1070 & 4.8 & 45 \\
\hline GP2 & Silva 2003 & Guarapuava -PR & $\mathrm{UM}$ & $25^{\circ} 21^{\prime}$ & $51^{\circ} 28^{\prime}$ & 1100 & 4.8 & 55 \\
\hline GP3 & Silva 2003 & Guarapuava -PR & MM & $25^{\circ} 21^{\prime}$ & $51^{\circ} 28^{\prime}$ & - & 4.8 & 42 \\
\hline IG & Ivanauskas et al. 1999 & Itatinga - SP & MS & $23^{\circ} 17^{\prime}$ & $48^{\circ} 33^{\prime}$ & 580 & 4.8 & 97 \\
\hline IT & Este estudo 2011 & Itaberá - SP & MM & $23^{\circ} 50^{\prime}$ & $49^{\circ} 08^{\prime}$ & 680 & 4.8 & 135 \\
\hline MC & Tomasulo \& Cordeiro 2000 & Mogi das Cruzes - SP & MD & - & - & 810 & 4.8 & 133 \\
\hline NP & Nascimento et al. 2001 & Nova Prata - RS & MM & $28^{\circ} 56^{\prime}$ & $51^{\circ} 53^{\prime}$ & 660 & 9.5 & 55 \\
\hline PI & Seger et al. 2005 & Pinhais - PR & MM & $25^{\circ} 24^{\prime}$ & $49^{\circ} 07^{\prime}$ & 900 & 5.0 & 41 \\
\hline PR & Ivanauskas \& Rodrigues 2000 & Piracicaba - SP & SDM & $22^{\circ} 39^{\prime}$ & $47^{\circ} 39^{\prime}$ & 554 & 4.8 & 110 \\
\hline RG1 & Nascimento 1994 & Ribeirão Grande - SP & MD & $24^{\circ} 16^{\prime}$ & $48^{\circ} 25^{\prime}$ & - & 4.8 & 23 \\
\hline RG2 & Nascimento 1994 & Ribeirão Grande - SP & MD & $24^{\circ} 16^{\prime}$ & $48^{\circ} 25^{\prime}$ & - & 4.8 & 52 \\
\hline RG3 & Nascimento 1994 & Ribeirão Grande - SP & MD & $24^{\circ} 16^{\prime}$ & $48^{\circ} 25^{\prime}$ & - & 4.8 & 33 \\
\hline SA1 & Mantovani et al. 1991 & Salesópolis - SP & MD & - & - & 850 & 4.8 & 29 \\
\hline SA2 & Mantovani et al. 1991 & Salesópolis - SP & MD & - & - & 830 & 4.8 & 39 \\
\hline SA3 & Mantovani et al. 1991 & Salesópolis - SP & MD & - & - & 840 & 4.8 & 42 \\
\hline SA4 & Mantovani et al. 1991 & Salesópolis - SP & MD & - & - & 850 & 4.8 & 46 \\
\hline SB1 & Dias 2004 & Sete Barras - SP & MD & $24^{\circ} 00^{\prime}$ & $47^{\circ} 55^{\prime}$ & - & 5.0 & 155 \\
\hline SB2 & Dias 2004 & Sete Barras - SP & MD & $24^{\circ} 00^{\prime}$ & $47^{\circ} 55^{\prime}$ & - & 5.0 & 157 \\
\hline SB3 & Aguiar 2003 & Sete Barras - SP & MD & $24^{\circ} 03^{\prime}$ & $47^{\circ} 59^{\prime}$ & 800 & 5.0 & 108 \\
\hline SB4 & Dias et al. 2000 & Sete Barras - SP & MD & $24^{\circ} 00^{\prime}$ & $47^{\circ} 45^{\prime}$ & - & 10.0 & 152 \\
\hline SC & Silva \& Soares 2002 & São Carlos - SP & MS & $21^{\circ} 55^{\prime}$ & $47^{\circ} 48^{\prime}$ & 850 & 5.0 & 77 \\
\hline SF1 & Sonego et al. 2007 & São Francisco de Paula - RS & $\mathrm{MM}$ & $29^{\circ} 27^{\prime}$ & $50^{\circ} 25^{\prime}$ & 923 & 5.0 & 41 \\
\hline SF2 & Sonego et al. 2007 & São Francisco de Paula - RS & $\mathrm{MM}$ & $29^{\circ} 27^{\prime}$ & $50^{\circ} 25^{\prime}$ & 923 & 10.0 & 41 \\
\hline SJ1 & Durigan 1999 & São João do Triunfo - PR & MM & $25^{\circ} 34^{\prime}$ & $50^{\circ} 05^{\prime}$ & 780 & 10.0 & 51 \\
\hline SJ2 & Sanqueta et al. 2002 & São João do Triunfo - PR & MM & $25^{\circ} 34^{\prime}$ & $50^{\circ} 05^{\prime}$ & 780 & 10.0 & 65 \\
\hline SJC & Silva 1989 & São José dos Campos - SP & MD & $23^{\circ} 12^{\prime}$ & $45^{\circ} 52$ & $640-1040$ & 4.8 & 195 \\
\hline
\end{tabular}


Table 1. Continuation.

\begin{tabular}{|c|c|c|c|c|c|c|c|c|}
\hline LC & Reference & Municipality - state & $\mathrm{CF}$ & Lat & Long & Alt & DBH & SR \\
\hline SL1 & Tabarelli et al. 1994 & São Luiz do Paraitinga - SP & $\mathrm{DM}$ & $24^{\circ} 21^{\prime}$ & $46^{\circ} 30^{\prime}$ & - & 3.2 & 36 \\
\hline SL2 & Tabarelli et al. 1994 & São Luiz do Paraitinga - SP & $\mathrm{MD}$ & $24^{\circ} 21^{\prime}$ & $47^{\circ} 30^{\prime}$ & - & 3.2 & 36 \\
\hline SM1 & Dias 1993 & São Miguel Arcanjo - SP & $\mathrm{MD}$ & - & - & 760 & 10.0 & 152 \\
\hline SM2 & Custodio Filho 2002 & São Miguel Arcanjo - SP & $\mathrm{MD}$ & $24^{\circ} 00^{\prime}$ & $48^{\circ} 00^{\prime}$ & 1000 & 10.0 & 69 \\
\hline SM3 & Custodio Filho 2002 & São Miguel Arcanjo - SP & $\mathrm{MD}$ & $24^{\circ} 00^{\prime}$ & $48^{\circ} 00^{\prime}$ & 800 & 10.0 & 78 \\
\hline SM4 & Rodrigues et al. 2004 & São Miguel Arcanjo - SP & DS & $24^{\circ} 00^{\prime}$ & $47^{\circ} 45^{\prime}$ & 350 & 5.0 & 205 \\
\hline SM5 & Lima \& Moura 2006 & São Miguel Arcanjo - SP & MD & $24^{\circ} 20^{\prime}$ & $47^{\circ} 44^{\prime}$ & $700-900$ & 4.8 & 156 \\
\hline SP & Baitello et al. 1992 & São Paulo - SP & $\mathrm{MD}$ & $23^{\circ} 22^{\prime}$ & $46^{\circ} 26^{\prime}$ & $850-1200$ & 10.0 & 140 \\
\hline SR & Leite \& Rodrigues 2008 & São Roque - SP & MS & $23^{\circ} 31^{\prime}$ & $47^{\circ} 06^{\prime}$ & $850-1025$ & 4.8 & 117 \\
\hline UB1 & Sanchez 1999 & Ubatuba - SP & $\mathrm{MD}$ & - & - & 600 & 4.8 & 106 \\
\hline UB2 & Sanchez 1999 & Ubatuba - SP & MD & - & - & 1000 & 4.8 & 91 \\
\hline
\end{tabular}

LC - location code; SP - São Paulo; PR - Paraná; SC - Santa Catarina; RS - Rio Grande do Sul; CF - class formation; MM - montane mixed rain forest; UM - upper montane mixed rain forest; $\mathrm{MD}$ - montane dense rain forest; SD - submontane dense rain forest; MS - montane semideciduous seasonal forest; MDS - montane deciduous seasonal forest; SR - species richness. Lat - latitude (south); Long. - longitude (west); Alt - Altitude (in m); DBH - Diameter at breast height (in cm), breast height being defined as $1.30 \mathrm{~m}$.

single species - $21(44.7 \%)$ of those in upper strata and 19 $(48.7 \%)$ of those in the lower strata. In the upper stratum, Moraceae, Myrtaceae, Rubiaceae, Meliaceae and Arecaceae accounted for $11.6 \%, 11.3 \%, 6.4 \%, 6.3 \%$, and $5.2 \%$ of the individuals sampled, respectively). The predominance of these families is largely due to high relative densities observed for Sorocea bonplandii (11.5\%), Eugenia ligustrina (6.1\%), Rudgea jasminoides (3.4\%), Trichilia catigua (3.1\%) and Syagrus romanzoffiana (3.7\%), respectively. In the lower stratum, Rubiaceae, Moraceae, Myrtaceae, Rutaceae and Meliaceae accounted for $32.2 \%, 13.5 \%, 11.5 \%, 6.8 \%$, and $5.0 \%$ of the individuals sampled, respectively. The species Psychotria suterella (19.1\% of the total), S. bonplandii (13.5\% of the total) and Helietta apiculata (4.5\% of the total) accounted for more than half of the individuals identified in their respective families (Rubiaceae, Moraceae and Rutaceae). In addition, Faramea montevidensis and Rudgea cf. jasminoides, respectively, accounted for $6.5 \%$ and $3.7 \%$ of the Rubiaceae identified, whereas Eugenia ramboi (4.75\%) was the most common Myrtaceae. Among the Meliaceae, no single species was predominant.

The most species-rich genera in the upper stratum were Ocotea and Nectandra (8 species and 3 species, respectively accounting for $66.7 \%$ and $25.0 \%$ of the Lauraceae species); Casearia (4 species, $66.7 \%$ of the Salicaceae); Eugenia and Myrcia (4 species each, both accounting for $33.3 \%$ of the Myrtaceae); Machaerium, (4 species, $66.7 \%$ of the Leguminosae); Trichilia (4 species, $66.7 \%$ of the Meliaceae); Solanum (3 species, $75.0 \%$ of the Solanaceae) and Zanthoxylum (3 species, $60.0 \%$ of the Rutaceae). In the lower stratum, these were Eugenia, Miconia and Trichilia (5 species, 5 species and 4 species, accounting for $50.0 \%, 83.3 \%$ and $80 \%$ of the Myrtaceae, Melastomataceae and Meliaceae, respectively). Therefore, only two of the nine genera that were prominent in the upper stratum were also prominent in the lower stratum.
Of the species sampled in both strata, nine are under some degree of threat regarding their conservation: Chionanthus filiformis, Ilex paraguariensis, Mollinedia argyrogyna and Solanum bullatum, all four of which are classified as being dependent on conservation measures (IUCN 1994); A. angustifolia, classified as vulnerable (SMA 2004), endangered (IBAMA 1993) or critically endangered (IUCN 2001); Euterpe edulis, classified as vulnerable (SMA 2004) or endangered (IBAMA 1993); Aspidosperma polyneuron and Cedrela fissilis, both classified as endangered (IUCN 1994); and Aspidosperma tomentosum, classified as endangered (SMA 2004). The occurrence of these species in the fragment studied underscores their ecological importance, contributing to the conservation of their populations, although E. edulis is not a native species, according to reports by former employees of the Station. Those former employees informed us that the species was deliberately introduced in the 1960s, and, after having established itself, expanded throughout the forest. It is noteworthy that the limiting the size of continuous protected areas can have a negative effect on the maintenance of species that are rare or have been overexploited in the past, especially those with intrinsic dispersion difficulty over long distances. It is known that many plant species in forests that are at an advanced successional stage produce large seeds or fruits that are in turn dispersed by a small number of larger animals, which also require well-maintained and relatively extensive continuous stretches of forest for their survival (Galetti et al. 2010). All species listed, with the exception of E. edulis and A. polyneuron, were represented by less than 10 individuals in each of the strata.

Rare species, i.e., those sampled from a single individual, accounted for 35 (25.9\%) of the species sampled in the upper stratum, one of those ( $S$. bullatum) being endangered. In the lower stratum, there were 26 such 
Tiago Maciel Ribeiro, Natália Macedo Ivanauskas, Sebastião Venâncio Martins, Rodrigo Trassi Polisel,

Rochelle Lima Ramos dos Santos and Aurino Miranda Neto

Table 2. List of woody species sampled in a fragment of mixed rain forest at the Itaberá Ecological Station, Itaberá, Brazil.

\begin{tabular}{|c|c|c|c|c|}
\hline \multirow{2}{*}{$\begin{array}{l}\text { FAMILY } \\
\text { Genus and species }\end{array}$} & \multirow{2}{*}{ Local name(s) } & \multirow{2}{*}{$\begin{array}{l}\text { Collector and } \\
\text { exsiccate }^{*}\end{array}$} & \multicolumn{2}{|c|}{ Stratum } \\
\hline & & & Upper** & Lower $^{* * *}$ \\
\hline \multicolumn{5}{|l|}{ ANACARDIACEAE } \\
\hline Astronium graveolens Jacq. & guaritá, aroeirão & & $\mathrm{X}$ & $\mathrm{X}$ \\
\hline Lithraea molleoides (Vell.) Engl. & aroeira-brava & & $\mathrm{X}$ & \\
\hline Tapirira guianensis Aubl. & peito-de-pombo & RLRS 140 & $\mathrm{X}$ & \\
\hline \multicolumn{5}{|l|}{ ANNONACEAE } \\
\hline Duguetia lanceolata A.St.-Hil. & pindaíva, corticeira & RLRS 141 & $\mathrm{X}$ & $\mathrm{x}$ \\
\hline Guatteria australis A.St.-Hil. & araticum & & $\mathrm{X}$ & $\mathrm{x}$ \\
\hline Porcelia macrocarpa (Warm.) R.E. Fr. & banana-de-macaco & & $\mathrm{X}$ & \\
\hline Xylopia brasiliensis Spreng. & Pindaíba & RTP 1001 & $\mathrm{X}$ & \\
\hline Xylopia langsdorfiana St.Hilaire \& Tulasne & Cedrinho & RTP 100 & & \\
\hline \multicolumn{5}{|l|}{ APOCYNACEAE } \\
\hline Aspidosperma polyneurum Müll.Arg. & peroba-rosa & & $\mathrm{X}$ & $\mathrm{X}$ \\
\hline Aspidosperma tomentosum Mart. & pereiro-do-campo & & $\mathrm{x}$ & $\mathrm{x}$ \\
\hline Tabernaemontana laeta Mart. & mata-pasto & & $\mathrm{X}$ & \\
\hline \multicolumn{5}{|l|}{ AQUIFOLIACEAE } \\
\hline Ilex dumosa Reissek & mate, erva-mate & RLRS 143 & & \\
\hline Ilex paraguariensis A.St.-Hil. & mate, erva-mate & & $\mathrm{x}$ & \\
\hline \multicolumn{5}{|l|}{ ARALIACEAE } \\
\hline Dendropanax cuneatus (DC.) Decne. \& Planch. & maria-mole & RLRS 53 & $\mathrm{x}$ & $\mathrm{x}$ \\
\hline Dendropanax monogynus (Vell.) Seem. & & RLRS 118 & & \\
\hline Schefflera angustissima (Marchal) Frodin & mandioqueira & & $\mathrm{x}$ & \\
\hline Schefflera calva (Cham.) Frodin \& Fiaschi & mandioqueiro & & $\mathrm{X}$ & \\
\hline \multicolumn{5}{|l|}{ ARAUCARIACEAE } \\
\hline Araucaria angustifolia (Bertol.) Kuntze & pinheiro-brasileiro & RLRS 68 & $\mathrm{X}$ & \\
\hline \multicolumn{5}{|l|}{ ARECACEAE } \\
\hline Euterpe edulis Mart. & palmito-juçara & & $\mathrm{x}$ & $\mathrm{x}$ \\
\hline Syagrus romanzoffiana (Cham.) Glassman & jerivá, coco-babão & & $\mathrm{X}$ & \\
\hline \multicolumn{5}{|l|}{ ASTERACEAE } \\
\hline Dasyphyllum brasiliense (Spreng.) Cabrera & guaiapá-parreira & & & $\mathrm{x}$ \\
\hline \multicolumn{5}{|l|}{ BIGNONIACEAE } \\
\hline Jacaranda macrantha Cham. & caroba & & $\mathrm{x}$ & $\mathrm{x}$ \\
\hline \multicolumn{5}{|l|}{ BORAGINACEAE } \\
\hline Cordia americana (L.) Gottschling \& J.S.Mill. & guajuvira & & $\mathrm{X}$ & $\mathrm{X}$ \\
\hline \multicolumn{5}{|l|}{ CANNABACEAE } \\
\hline Celtis iguanaea (Jacq.) Sarg. & gumbixava & & & $\mathrm{X}$ \\
\hline Trema micrantha (L.) Blume & crindiúva & & $\mathrm{x}$ & \\
\hline \multicolumn{5}{|l|}{ CARDIOPTERIDACEAE } \\
\hline Citronella paniculata (Mart.) R.A. Howard & pau-de-corvo & & $\mathrm{X}$ & $\mathrm{x}$ \\
\hline \multicolumn{5}{|l|}{ CARICACEAE } \\
\hline Jacaratia heptaphylla (Vell.) A. DC. & jaracatiá & & $\mathrm{X}$ & \\
\hline \multicolumn{5}{|l|}{ CELASTRACEAE } \\
\hline Maytenus aquifolia Mart. & espinheira-santa & & $\mathrm{X}$ & $\mathrm{X}$ \\
\hline
\end{tabular}


Table 2. Continuation

\begin{tabular}{|c|c|c|c|c|}
\hline \multirow{2}{*}{$\begin{array}{l}\text { FAMILY } \\
\text { Genus and species }\end{array}$} & \multirow{2}{*}{ Local name(s) } & \multirow{2}{*}{$\begin{array}{l}\text { Collector and } \\
\text { exsiccate }^{*}\end{array}$} & \multicolumn{2}{|c|}{ Stratum } \\
\hline & & & Upper ${ }^{* *}$ & Lower $^{* * *}$ \\
\hline Maytenus evonymoides Reissek & & & & $\mathrm{X}$ \\
\hline \multicolumn{5}{|l|}{ CLETHRACEAE } \\
\hline Clethra scabra Pers. & peroba-café & & $\mathrm{X}$ & \\
\hline \multicolumn{5}{|l|}{ COMBRETACEAE } \\
\hline Terminalia triflora (Griseb.) Lillo & capitãozinho & & $\mathrm{X}$ & \\
\hline \multicolumn{5}{|l|}{ CYATHEACEAE } \\
\hline Cyathea atrovirens (Langsd. \& Fisch.) Domin & samambaiaçú & & $\mathrm{x}$ & \\
\hline \multicolumn{5}{|l|}{ ELAEOCARPACEAE } \\
\hline Sloanea lasiocoma K. Schum. & sapopema & & $\mathrm{x}$ & $\mathrm{X}$ \\
\hline Sloanea monosperma Vell. & sapopema & & & $\mathrm{X}$ \\
\hline \multicolumn{5}{|l|}{ EUPHORBIACEAE } \\
\hline Acalypha gracilis Spreng. & & RLRS 142 & & \\
\hline Actinostemon concepcionis (Chodat \& Hassl.) Hochr. & folha-fedorenta & RLRS 71 & $\mathrm{x}$ & $\mathrm{X}$ \\
\hline Alchornea glandulosa Poepp. & tapiá, pau-jangada & & $\mathrm{X}$ & \\
\hline Alchornea triplinervia (Spreng.) Müll.Arg. & canela-raposa & & $\mathrm{X}$ & \\
\hline Croton floribundus Spreng. & capixingui & & $\mathrm{X}$ & $\mathrm{X}$ \\
\hline Sebastiania klotzschiana Müll.Arg. & branquinho & & $\mathrm{x}$ & $\mathrm{X}$ \\
\hline Tetrorchidium rubrivenium Poepp. & canemaçu & & $\mathrm{X}$ & \\
\hline \multicolumn{5}{|l|}{ FABACEAE - CAESALPINIOIDEAE } \\
\hline Cassia leptophylla Vogel & falso-barbatimão & & $\mathrm{X}$ & \\
\hline Copaifera langsdorffii Desf. & copaíba, pau-d'óleo & RLRS 54 & $\mathrm{x}$ & $\mathrm{X}$ \\
\hline Copaifera trapezifolia Hayne & & & $\mathrm{X}$ & $\mathrm{X}$ \\
\hline Holocalyx balansae Micheli & alecrim-de-campinas & & & $\mathrm{X}$ \\
\hline \multicolumn{5}{|l|}{ FABACEAE - MIMOSOIDEAE } \\
\hline Inga marginata Willd & ingá-feijão & & $\mathrm{X}$ & $\mathrm{X}$ \\
\hline Inga sessilis (Vell.) Mart. & ingá-ferradura & RLRS 83 & & $\mathrm{X}$ \\
\hline Inga striata Benth. & ingá-banana & & $\mathrm{x}$ & \\
\hline Parapiptadenia rigida (Benth.) Brenan & angico-cedro & & $\mathrm{x}$ & $\mathrm{X}$ \\
\hline Piptadenia gonoacantha (Mart.) J.F.Macbr. & pau-Jacaré & RLRS 55/120 & $\mathrm{x}$ & $\mathrm{X}$ \\
\hline Senegalia polyphylla (DC.) Britton \& Rose & monjoleiro & RLRS 79 & $\mathrm{x}$ & \\
\hline \multicolumn{5}{|l|}{ FABACEAE - FABOIDEAE } \\
\hline Centrolobium tomentosum Guillem. ex Benth. & araribá & RLRS 130 & & \\
\hline Dalbergia frutescens (Vell.) Briton & & & & $\mathrm{X}$ \\
\hline Exostyles godoyensis Soares-Silva \& Mansano & & RLRS 93; RTP 1009 & $\mathrm{X}$ & $\mathrm{X}$ \\
\hline Lonchocarpus subglaucescens Mart. ex Benth. & embira-de-sapo & RLRS 155 & $\mathrm{x}$ & \\
\hline Machaerium nyctitans (Vell.) Benth. & bico-de-pato & & $\mathrm{X}$ & $\mathrm{X}$ \\
\hline Machaerium paraguariense Hassl. & jacarandá-branco & & $\mathrm{x}$ & \\
\hline Machaerium scleroxylon Tul. & caviúna & & $\mathrm{X}$ & \\
\hline Machaerium stipitatum (DC.) Vogel & sapuvinha & & $\mathrm{X}$ & $\mathrm{X}$ \\
\hline Machaerium vestitum Vogel & cateretê & RLRS 85 & & \\
\hline \multicolumn{5}{|l|}{ LAURACEAE } \\
\hline Endlicheria paniculata (Spreng.) J.F.Macbr. & canela-peluda & TMR 13 & & $\mathrm{X}$ \\
\hline
\end{tabular}


Table 2. Continuation

\begin{tabular}{|c|c|c|c|c|}
\hline \multirow{2}{*}{$\begin{array}{l}\text { FAMILY } \\
\text { Genus and species }\end{array}$} & \multirow{2}{*}{ Local name(s) } & \multirow{2}{*}{$\begin{array}{l}\text { Collector and } \\
\text { exsiccate }^{\star}\end{array}$} & \multicolumn{2}{|c|}{ Stratum } \\
\hline & & & Upper** & Lower $^{* * *}$ \\
\hline Nectandra lanceolata Nees & canela-amarela & & $\mathrm{X}$ & \\
\hline Nectandra megapotamica (Spreng.) Mez & canela-imbuia & RLRS 77 & $\mathrm{X}$ & \\
\hline Nectandra oppositifolia Nees \& Mart. & canela-amarela & RLRS 119 & $\mathrm{X}$ & $\mathrm{X}$ \\
\hline Ocotea bicolor Vattimo-Gil & canela-fedida & & $\mathrm{X}$ & \\
\hline Ocotea corymbosa (Meisn.) Mez & canela-fedorenta & & $\mathrm{X}$ & \\
\hline Ocotea diospyrifolia (Meisn.) Mez & & RLRS 57 & $\mathrm{X}$ & \\
\hline Ocotea elegans $\mathrm{Mez}$ & canela-parda & RLRS 78 & $\mathrm{X}$ & $\mathrm{X}$ \\
\hline Ocotea indecora (Schott) Mez & & & $\mathrm{X}$ & $\mathrm{X}$ \\
\hline Ocotea pulchella (Nees \& Mart.) Mez & canela-lageana & RLRS112/116/123 & $\mathrm{X}$ & \\
\hline Ocotea silvestris Vattimo-Gil & canela & RLRS 48/TMR 15 & $\mathrm{X}$ & \\
\hline Ocotea sp. & & & $\mathrm{X}$ & \\
\hline Persea willdenovii Kosterm. & & RTP 1002 & $\mathrm{X}$ & \\
\hline \multicolumn{5}{|l|}{ LAXMANNIACEAE } \\
\hline Cordyline spectabilis Kunth \& C.D.Bouché & guaraíva & & $\mathrm{X}$ & $\mathrm{X}$ \\
\hline \multicolumn{5}{|l|}{ LECYTHIDACEAE } \\
\hline Cariniana estrellensis (Raddi) Kuntze & jequitibá-rosa & & $\mathrm{X}$ & \\
\hline \multicolumn{5}{|l|}{ LOGANIACEAE } \\
\hline Strychnos brasiliensis Mart. & estralo & & & $\mathrm{X}$ \\
\hline \multicolumn{5}{|l|}{ MALVACEAE } \\
\hline Ceiba speciosa (A.St.-Hil.) Ravenna & paineira & RLRS 156 & $\mathrm{X}$ & $\mathrm{X}$ \\
\hline Guazuma ulmifolia Lam. & araticum-bravo & & $\mathrm{X}$ & \\
\hline Luehea divaricata Mart. & açoita-cavalo & RTP $108 / 41$ & $\mathrm{X}$ & \\
\hline \multicolumn{5}{|l|}{ MELASTOMATACEAE } \\
\hline Miconia doriana Cogn. & & RLRS 98 & $\mathrm{X}$ & $\mathrm{X}$ \\
\hline Miconia hymenonervia (Raddi) Cogn. & & & & $\mathrm{X}$ \\
\hline Miconia latecrenata (DC.) Naudin & & & & $\mathrm{X}$ \\
\hline Miconia petropolitana Cogn. & jacatirão-mirim & RLRS 39 & $\mathrm{X}$ & $\mathrm{X}$ \\
\hline Miconia rigidiuscula Cogn. & & & & $\mathrm{X}$ \\
\hline Miconia tristis Spring & & & & $\mathrm{X}$ \\
\hline \multicolumn{5}{|l|}{ MELIACEAE } \\
\hline Cabralea canjerana (Vell.) Mart. & canjerana & RLRS $162 / 158$ & $\mathrm{X}$ & \\
\hline Cedrela fissilis Vell. & cedro-rosa & & $\mathrm{x}$ & \\
\hline Guarea macrophylla Vahl & marinheiro & RLRS 56 & & $\mathrm{X}$ \\
\hline Trichilia catigua A.Juss. & catiguá & RTP 1008 & $\mathrm{X}$ & $\mathrm{X}$ \\
\hline Trichilia claussenii C. DC. & catiguá-vermelho & RLRS 159 & $\mathrm{X}$ & $\mathrm{X}$ \\
\hline Trichilia elegans A.Juss. & canela-do-mato & RLRS 160 , TMR 5 & $\mathrm{X}$ & $\mathrm{X}$ \\
\hline Trichilia pallida Sw. & catiguá, marinheiro & TMR21 & $\mathrm{X}$ & $\mathrm{X}$ \\
\hline \multicolumn{5}{|l|}{ MONIMIACEAE } \\
\hline Mollinedia argyrogyna Perkins & corticeira & RLRS 72 & $\mathrm{X}$ & \\
\hline Mollinedia clavigera Tul. & cidreira-do-mato & RLRS 94 & & \\
\hline Mollinedia elegans Tul. & & RLRS 88 & $\mathrm{X}$ & \\
\hline Mollinedia schottiana (Spreng.) Perkins & guatambú-langanha & & & $\mathrm{X}$ \\
\hline
\end{tabular}


Table 2. Continuation

\begin{tabular}{|c|c|c|c|c|}
\hline \multirow{2}{*}{$\begin{array}{l}\text { FAMILY } \\
\text { Genus and species }\end{array}$} & \multirow{2}{*}{ Local name(s) } & \multirow{2}{*}{$\begin{array}{l}\text { Collector and } \\
\text { exsiccate }^{*}\end{array}$} & \multicolumn{2}{|c|}{ Stratum } \\
\hline & & & Upper** & Lower $^{* * *}$ \\
\hline Mollinedia uleana Perkins & & RLRS 87 & & $\mathrm{x}$ \\
\hline \multicolumn{5}{|l|}{ MORACEAE } \\
\hline Ficus insipida Willd. & mata-pau & RLRS95,TMR17 & & \\
\hline Ficus luschnathiana (Miq.) Miq. & gameleira-vermelha & TMR 14 & $\mathrm{x}$ & \\
\hline Sorocea bonplandii (Baill.) W.C.Burger, Lanj. \& de Boer & falsa-espinheira-santa & RLRS 63/59 & $\mathrm{X}$ & $\mathrm{x}$ \\
\hline \multicolumn{5}{|l|}{ MYRSINACEAE } \\
\hline Rapanea loefgrenii Mez & pororoca & RLRS 38/96 & $\mathrm{X}$ & $\mathrm{x}$ \\
\hline Rapanea umbellata (Mart.) Mez & capororocão & TMR 7 & $\mathrm{X}$ & \\
\hline \multicolumn{5}{|l|}{ MYRTACEAE } \\
\hline Calyptranthes concinna DC. & guamirim-de-facho & TMR 8 & & $\mathrm{X}$ \\
\hline Calyptranthes grandifolia O.Berg & guamirim-chorão & & $\mathrm{x}$ & \\
\hline Campomanesia guazumifolia (Cambess.) O.Berg & sete-capotes & & $\mathrm{X}$ & $\mathrm{X}$ \\
\hline Campomanesia xanthocarpa (Mart.) O.Berg & guabirobeira & & $\mathrm{x}$ & $\mathrm{X}$ \\
\hline Eugenia dodonaeifolia Cambess. & & & & $\mathrm{x}$ \\
\hline Eugenia hiemalis Cambess. & guamirim-miúdo & RTP 1000 & & \\
\hline Eugenia ligustrina (Sw.) Willd & & RLRS 60, RTP 1000 & $\mathrm{x}$ & \\
\hline Eugenia ramboi D.Legrand & batinga-branca & RTP 997 & $\mathrm{X}$ & $\mathrm{x}$ \\
\hline Eugenia stenophylla O.Berg & & & $\mathrm{X}$ & $\mathrm{x}$ \\
\hline Eugenia uniflora $\mathrm{L}$. & pitangueira & RTP 998 & $\mathrm{x}$ & $\mathrm{x}$ \\
\hline Myrcia laruotteana Cambess. & cambuí & TMR 6 & $\mathrm{x}$ & \\
\hline Myrcia multiflora (Lam). DC. & cambuí & & $\mathrm{X}$ & \\
\hline Myrcia pulchra (O.Berg) Kiaersk & & & $\mathrm{X}$ & \\
\hline Myrcia rostrata DC. & guamirim-miúdo & & $\mathrm{X}$ & $\mathrm{x}$ \\
\hline Myrciaria floribunda (H.West ex Willd.) O.Berg & camboim & & & $\mathrm{x}$ \\
\hline Myrciaria rivularis (Cambess.) O.Berg & & & $\mathrm{x}$ & \\
\hline Myrciaria tenella (DC.) O.Berg & cambuí & RLRS 50 & & \\
\hline Psidium sp. & & & & $\mathrm{X}$ \\
\hline \multicolumn{5}{|l|}{ NYCTAGINACEAE } \\
\hline Guapira opposita (Vell.) Reitz & maria-mole & RLRS 179 & $\mathrm{X}$ & $\mathrm{X}$ \\
\hline \multicolumn{5}{|l|}{ OLEACEAE } \\
\hline Chionanthus filiformis (Vell.) P.S.Green & pitaguará & RLRS 69 & $\mathrm{x}$ & $\mathrm{X}$ \\
\hline \multicolumn{5}{|l|}{ OPILIACEAE } \\
\hline Agonandra brasiliensis Miers ex Benth. \& Hook.f. & mamica-de-cadela & & $\mathrm{X}$ & \\
\hline \multicolumn{5}{|l|}{ PERACEAE } \\
\hline Pera glabrata (Schott) Poepp. ex Baill. & tabocuva & TMR 11 & $\mathrm{x}$ & \\
\hline \multicolumn{5}{|l|}{ PHYTOLACCACEAE } \\
\hline Seguieria langsdorffii Moq. & laranja-do-mato & & $\mathrm{x}$ & \\
\hline \multicolumn{5}{|l|}{ PIPERACEAE } \\
\hline Piper arboreum Aubl. & & RTP 1011 & & $\mathrm{x}$ \\
\hline Piper cf. bowiei Yunck. & & & & $\mathrm{X}$ \\
\hline \multicolumn{5}{|l|}{ PROTEACEAE } \\
\hline Roupala brasiliensis Klotzsch & carvalho-brasileiro & & & $\mathrm{X}$ \\
\hline
\end{tabular}


Table 2. Continuation

\begin{tabular}{|c|c|c|c|c|}
\hline \multirow{2}{*}{$\begin{array}{l}\text { FAMILY } \\
\text { Genus and species }\end{array}$} & \multirow{2}{*}{ Local name(s) } & \multirow{2}{*}{$\begin{array}{c}\text { Collector and } \\
\text { exsiccate }\end{array}$} & \multicolumn{2}{|c|}{ Stratum } \\
\hline & & & Upper $^{* *}$ & Lower ${ }^{* * *}$ \\
\hline \multicolumn{5}{|l|}{ RHAMNACEAE } \\
\hline Colubrina glandulosa Perkins & saguraji & & $\mathrm{x}$ & \\
\hline Rhamnidium elaeocarpum Reissek & saguaraji-amarelo & & $\mathrm{x}$ & $\mathrm{X}$ \\
\hline \multicolumn{5}{|l|}{ ROSACEAE } \\
\hline Prunus myrtifolia (L.) Urb. & pessegueiro bravo & & $\mathrm{X}$ & $\mathrm{X}$ \\
\hline \multicolumn{5}{|l|}{ RUBIACEAE } \\
\hline Alibertia concolor (Cham.) K.Schum. & & & $\mathrm{x}$ & \\
\hline Alibertia myrciifolia Spruce ex K.Schum. & marmemelinho & & $\mathrm{x}$ & $\mathrm{X}$ \\
\hline Amaioua intermedia Mart. ex Schult. \& Schult.f. & café-de-bugre & & $\mathrm{x}$ & $\mathrm{X}$ \\
\hline Chomelia obtusa Cham. \& Schltdl. & & & $\mathrm{x}$ & \\
\hline Faramea montevidensis (Cham. \& Schltdl.) DC. & & RTP 1007 & $\mathrm{x}$ & $\mathrm{X}$ \\
\hline Ixora venulosa Benth. & ixora & RLRS 174 & $\mathrm{x}$ & $\mathrm{X}$ \\
\hline Psychotria longipes Müll.Arg. & & RLRS 147 & & \\
\hline Psychotria stachyoides Benth. & & & & $\mathrm{X}$ \\
\hline Psychotria suterella Müll.Arg. & cafezinho-roxo & & $\mathrm{x}$ & $\mathrm{X}$ \\
\hline Rudgea gardenioides (Cham.) Müll.Arg. & arapoca & RLRS 146 & & \\
\hline Rudgea jasminoides (Cham.) Müll.Arg. & jasmim-do-mato & RLRS 148/61 & $\mathrm{x}$ & $\mathrm{X}$ \\
\hline Rudgea cf. jasminoides (Cham.) Müll.Arg. & jasmim-do-mato & & & $\mathrm{x}$ \\
\hline \multicolumn{5}{|l|}{ RUTACEAE } \\
\hline Esenbeckia febrifuga (A.St.-Hil.) A.Juss. ex Mart. & chupa-ferro & RLRS 99 & & \\
\hline Helietta apiculata Benth. & canela-de-veado & TMR16 & $\mathrm{x}$ & $\mathrm{x}$ \\
\hline Pilocarpus pennatifolius Lem. & crista-de-peru & RLRS 101 & $\mathrm{X}$ & $\mathrm{X}$ \\
\hline Zanthoxylum caribaeum Lam. & arruda-brava & & $\mathrm{x}$ & \\
\hline Zanthoxylum fagara (L.) Sarg. & mamica-de-porca & & $\mathrm{X}$ & \\
\hline Zanthoxylum monogynum A.St.-Hil. & maminha-de-porca & & $\mathrm{X}$ & $\mathrm{x}$ \\
\hline \multicolumn{5}{|l|}{ SALICACEAE } \\
\hline Casearia decandra Jacq. & guassatonga & & $\mathrm{x}$ & $\mathrm{x}$ \\
\hline Casearia gossypiosperma Briq. & pau-de-espeto & & $\mathrm{x}$ & \\
\hline Casearia obliqua Spreng. & & & $\mathrm{X}$ & \\
\hline Casearia sylvestris $\mathrm{Sw}$. & café-do-mato & & $\mathrm{x}$ & \\
\hline Prockia crucis P. Browne ex $\mathrm{L}$. & marmeladinha & RTP 995 & $\mathrm{X}$ & $\mathrm{X}$ \\
\hline Xylosma tweediana (Clos) Eichler & espinho-de-judeu & & $\mathrm{x}$ & \\
\hline \multicolumn{5}{|l|}{ SAPINDACEAE } \\
\hline Allophylus edulis (A.St.-Hil. et al.) Hieron. ex Niederl. & três-folhas-do-mato & & $\mathrm{x}$ & $\mathrm{x}$ \\
\hline Cupania tenuivalvis Radlk. & camboatá-miúdo & RLRS 45 & $\mathrm{x}$ & $\mathrm{x}$ \\
\hline Cupania vernalis Cambess. & camboatá-vermelho & RLRS 42 & $\mathrm{X}$ & $\mathrm{X}$ \\
\hline Diatenopteryx sorbifolia Radlk. & maria-preta & TMR 12 & $\mathrm{x}$ & \\
\hline Matayba elaeagnoides Radlk. & camboatá-branco & RTP 1005 & $\mathrm{X}$ & $\mathrm{X}$ \\
\hline \multicolumn{5}{|l|}{ SAPOTACEAE } \\
\hline Chrysophyllum gonocarpum (Mart. \& Eichler ex Miq.) Engl. & aguaí-da-serra & & $\mathrm{X}$ & $\mathrm{X}$ \\
\hline Chrysophyllum marginatum (Hook. \& Arn.) Radlk. & aguaí-vemelho & & $\mathrm{X}$ & \\
\hline Pouteria gardneriana (A.DC.) Radlk. & aguaí-guaçu & & $\mathrm{X}$ & \\
\hline
\end{tabular}


Table 2. Continuation.

\begin{tabular}{|c|c|c|c|c|}
\hline \multirow{2}{*}{$\begin{array}{l}\text { FAMILY } \\
\text { Genus and species }\end{array}$} & \multirow{2}{*}{ Local name(s) } & \multirow{2}{*}{$\begin{array}{l}\text { Collector and } \\
\text { exsiccate }^{*}\end{array}$} & \multicolumn{2}{|c|}{ Stratum } \\
\hline & & & Upper $^{* *}$ & Lower ${ }^{* * *}$ \\
\hline \multicolumn{5}{|l|}{ SOLANACEAE } \\
\hline Brunfelsia pauciflora (Cham. \& Schltdl.) Benth. & manacá & & & $\mathrm{X}$ \\
\hline Cestrum intermedium Sendtn. & coerana & & $\mathrm{x}$ & $\mathrm{x}$ \\
\hline Solanum argenteum Dunal & & & & $\mathrm{x}$ \\
\hline Solanum bullatum Vell. & joá-açu & RLRS 121/126/166 & $\mathrm{X}$ & \\
\hline Solanum pseudoquina A.St.-Hil. & falsa-quina & RLRS 132 & & \\
\hline Solanum sanctae-catharinae Dunal & joá-manso, Juá & RLRS 167 & $\mathrm{X}$ & $\mathrm{x}$ \\
\hline Solanum swartzianum Roem. \& Schult. & & TMR 1 & $\mathrm{x}$ & $\mathrm{x}$ \\
\hline \multicolumn{5}{|l|}{ STYRACACEAE } \\
\hline Styrax acuminatus Pohl & jacutinga & RLRS 163 & & \\
\hline Styrax camporum Pohl & benjoeiro & & $\mathrm{X}$ & \\
\hline Styrax pohlii A.DC. & árvore-de-bálsamo & & $\mathrm{x}$ & \\
\hline \multicolumn{5}{|l|}{ URTICACEAE } \\
\hline Cecropia glaziovii Snethl. & embaúba-vermelha & & $\mathrm{X}$ & \\
\hline Cecropia pachystachya Trécul & embaúba-do-brejo & & $\mathrm{x}$ & \\
\hline \multicolumn{5}{|l|}{ VERBENACEAE } \\
\hline Verbenaceae 1 & & TMR 29 & & $\mathrm{X}$ \\
\hline \multicolumn{5}{|l|}{ VOCHYSIACEAE } \\
\hline Vochysia magnifica Warm. & cinzeiro-da-serra & & $\mathrm{X}$ & \\
\hline Vochysia tucanorum Mart. & pau-de-tucano & TMR $18 / 19$ & & \\
\hline
\end{tabular}

${ }^{\star}$ Initials of the collector and the accession number(s) of the exsiccate(s) deposited at the Dom Bento Pickel Herbarium (code, SPSF); ${ }^{*}$ including individuals with a diameter at breast height $\geq 15 \mathrm{~cm} ;{ }^{* * *}$ including individuals with a diameter at breast height $<15 \mathrm{~cm}$ and an overall height $\geq 30 \mathrm{~cm}$.

species ( $28 \%$ of the species sampled), one of which ( $A$. tomentosum) is endangered.

\section{Diversity}

For the upper stratum, we estimated the $\mathrm{H}^{\prime}$ at 4.12 (4.11 $<\mathrm{H}^{\prime}<4.30$, using 95\% jackknife confidence intervals). The community showed low dominance, as evidenced by the estimated J' and $\mathrm{MC}$ values (0.84 and 1:10.51, respectively). All of these values are higher than those typically found for stretches of Araucaria forest in the state of São Paulo and in southern Brazil in general. Using the same methodology, Souza (2008) estimated H' and J' at 3.08 and 0.73 , respectively, for an area near the city of Campos do Jordão (in the state of São Paulo), compared with 3.81 and 0.70, respectively, for an area that had been regenerating for the last 120 years near the city of Barra do Chapéu (also in the state of São Paulo). Also in the Campos do Jordão area, Los (2004) evaluated six discontinuous blocks of 0.25 ha each and reported an H' of 3.43. For southern Brazil, $H$ ' values found in the literature vary widely, ranging from 2.76 (Rondon-Neto et al. 2002) to 3.67 or higher (Reginato \& Goldenberg 2007). Unfortunately, differences in sampling methodology, historical uses of areas and landscape conditions, as well as other factors, complicate comparisons across studies. In the lower stratum, these values were also high, although less dramatically so: $\mathrm{H}^{\prime}=3.5\left(3.46<\mathrm{H}^{\prime}<3.72\right.$, using $95 \%$ jackknife confidence intervals); J' $=0.77$; and $\mathrm{MC}=1: 8.06$. Souza (2008) also highlighted the great diversity of this stratum near Campos do Jordão ( $\mathrm{H}$ ' and J' of 3.81 and 0.84 , respectively) and Barra do Chapéu (3.44 and 0.79, respectively). Unfortunately, the lower strata of Araucaria forests have been poorly studied, resulting in a scarcity of studies for comparisons, and the limitations mentioned above for the upper stratum also apply here. Nevertheless, the fact that high diversity was also detected in the lower stratum underscores the importance of the area for conservation and hence the importance of proper management of this regeneration, so that the values identified are maintained or increased.

The confidence intervals estimated for $\mathrm{H}^{\prime}$ indicate that the upper stratum contains greater species diversity (alpha) than does the lower stratum, although this index is influenced by the number of individuals sampled (Durigan 2009).

The high diversity observed in both strata at the Station is likely due to its geographical position, inserted into an area of ecological tension. On Guapiara Plateau, where the Station is located, in addition to the mixed rain forest, other forest settings occur, ranging from dense rain forest, near the Paranapiacaba mountain range, to stretches of savanna 
(cerrado) and semideciduous seasonal forest further inland (Ab'Saber 2003). According to Jarenkow \& Budke (2009), the floristic richness of Araucaria forests decreases with increasing latitude, being greater in areas of contact with other forest formations, in which cases the araucaria plays a secondary role in forest structure.

\section{Floristic similarity}

The upper and lower strata were very similar floristically (with Jaccard and Sorensen indices of 0.40 and 0.57 , respectively), having 65 species in common. More than two thirds of the lower stratum species also occurred in the upper stratum. However, a little over half the species present in the upper stratum were not sampled in the lower stratum. Therefore, pronounced floristic changes in the area are expected in the long term, with likely physiognomic changes associated to it, chiefly because of local extinction of its typical emerging species, A. angustifolia, not sampled in lower stratum.

Comparing our survey with that of a remaining stretch of the same formation in Campos do Jordão (Souza, 2008), we observed low floristic affinity between the areas (Jaccard and Sorensen indices of 0.08 and 0.15, respectively, considering the species sampled in both strata), with only 18 species in common. Even if we consider only those individuals identified down to the species level, the figures do not change. Analyzing only the upper stratum, the indices are even lower ( 0.05 and 0.10 , respectively), even if we consider only the individuals identified down to the species level (0.06 and 0.11 , respectively), with only 10 species in common. The same goes for the lower component (0.06 and 0.11 , respectively, for all species, compared with 0.06 and 0.12 , respectively, for only those identified down to the species level), with only eight species in common. The low floristic similarity observed might be explained by the natural disjunction between areas (approximately 390 $\mathrm{km}$ away in a straight line, $22^{\circ} 41^{\prime} 30^{\prime \prime} \mathrm{S} ; 4^{\circ} 27^{\prime} 52^{\prime \prime} \mathrm{W}$ ), given that this formation begins to become naturally fragmented in the southern part of the state of São Paulo, recurring at higher altitudes in the Serra do Mar and Mantiqueira mountain ranges. In addition, the Itaberá stretch is strongly influenced by neighboring forest formations, which results in it being mischaracterized floristically as a typical remnant of Araucaria forest, according to the latter approach (considering only specimens identified down to the species level). Another factor would be the distinct conditions of the landscape, particularly in terms of the extent of continuous protected area, given the previously mentioned effects that it can have on the flora.

The Itaberá Ecological Station most resembles a remnant of Araucaria forest in its most proximate regions, on the slopes of the Serra de Paranapiacaba mountain range (approximately $70 \mathrm{~km}$ away in a straight line, at $24^{\circ} 28^{\prime} \mathrm{S}$; $49^{\circ} 01^{\prime} \mathrm{W}$ ), an area also evaluated by Souza (2008), which has been regenerating for the last 120 years near the municipality of Barra do Chapéu. Considering both strata, there is a certain floristic affinity between the areas (Jaccard and Sorensen indices of 0.20 and 0.34 , respectively), with 53 species in common. However, these cannot be considered floristically similar, even if we consider only the species identified down to the species level, with Jaccard and Sorensen indices of 0.22 and 0.37 , respectively. In the upper stratum, the Jaccard and Sorensen indices were 0.21 and 0.35 , respectively, for all species, compared with 0.23 and 0.37 , respectively, for only those identified down to the species level, with 45 species in common. The greatest floristic similarity was observed in the lower stratum (Jaccard and Sorensen indices of 0.27 and 0.43 , respectively, for all species, compared with 0.31 and 0.48 , respectively, for only those identified down to the species level), with 36 species in common. Therefore, the lower strata of the two areas are floristically similar, according to the Jaccard index for all species sampled and according to both indices for only those identified down to the species level. Therefore, it is expected that, over the long term, there will be an increase in similarity between the upper strata of the two areas.

\section{Cluster analysis}

The matrix compiled from floristic listings comprised 1095 species, reflecting the floristic heterogeneity among the 53 areas considered. The most common species in the mixed rain forest were as follows: A. angustifolia (100\%); Campomanesia xanthocarpa (90\%); Casearia decandra (90\%); Ocotea puberula (75\%); Jacaranda puberula (75\%); Allophylus edulis (75\%); Matayba elaeagnoides (75\%); Rapanea umbellata (75\%); Sapium glandulatum (70\%); Sebastiania commersoniana (70\%); I. paraguariensis (70\%) Ocotea pulchella (70\%); Vernonia discolor (65\%); Styrax leprosus (65\%); Ilex theezans (60\%); Prunus myrtifolia (60\%); Cedrella fissilis (60\%); Clethra scabra (55\%) and Schinus terebinthifolius (50\%). Of those, only A. angustifolia is considered to be exclusive to Araucaria forests (Stehmann 2009), although C. xanthocarpa, S. commersoniana and $S$. leprosus have also been identified only in such forests. Jarenkow \& Budke (2009), in a review of floristic patterns in this formation, also demonstrated the high frequency of most of these species (above $80 \%$ in the 38 surveys evaluated), the exceptions being O. puberula, J. puberula, $O$. pulchella, I. theezans and C. scabra.

Considering the four areas of Araucaria forest in the state of São Paulo, we noted that they have only nine species in common: A. angustifolia, Cabralea canjerana, C. decandra, Guatteria australis, Myrcia fallax, Ocotea bicolor, R. umbellata, $R$. jasminoides and Solanum swartzianum. Of those, $C$. canjerana, C. decandra, G. australis, M. fallax and R. umbellata have also been shown to occur in $\geq 50 \%$ of the areas of dense rain forest evaluated in the state. In addition, $M$. fallax has been identified in semideciduous seasonal forests 
within the state, indicating its wide geographic distribution.

For the mixed rain forest evaluated in the present study, diversity $\left(\mathrm{H}^{\prime}\right)$ varied considerably, from 2.2 to 4.12 . However, methodological differences between surveys, some of which have used different indices or none at all, hinder comparisons across studies.

As can be seen in the dendrogram obtained from the cluster analysis (Fig. 2), there were four major groups, one survey, conducted in Ubatuba (Sanchez 1999), standing apart. The cophenetic correlation coefficient obtained is considered high, showing that the groups formed are consistent; that is, the results are robust and reliable.

The first group comprises the majority of surveys conducted in the dense rain forest in the state of São Paulo (Serra do Mar and Serra de Paranapiacaba mountain ranges). We noticed that the areas evaluated near the municipalities of Sete Barras, São Miguel Arcanjo and Capão Bonito had greater floristic affinity, possibly due to their geographical proximity, thus forming a more cohesive subgroup. The most common species in that group were C. canjerana, Alchornea triplinervia, Guapira opposita, Casearia sylvestris, C. decandra, Tapirira guianensis, Annona neosericea, Maytenus robusta, Endlicheria paniculata, S. bonplandii, Myrcia splendens and Amaioua intermedia, all occurring in at least half of the areas considered.

The second major group comprised surveys conducted in areas of dense rain forest in southern Brazil and near Campos do Jordão. The segregation of the two surveys conducted in the state of São Paulo is notable and probably reflects the effect of the natural geographic disconnect within the formation. The indicator species in this subgroup were Ilex taubertiana, Baccharis oreophila, Piptocarpha macropoda, O. bicolor, Ocotea glaziovii, Persea willdenovii, Calyptranthes lucida, Symplocos falcata and R. jasminoides. Jarenkow \& Budke (2009) point out that these areas have many species typical of upper montane environments, especially the Melastomataceae of the Miconia and Tibouchina genera, increasing intragroup dissimilarity. However, there were no Tibouchina spp. sampled in either of the two areas considered.

The third group comprised three surveys conducted near the municipality of Ribeirão Grande (in the state of São Paulo), in the dense rain forest of the Serra de Paranapiacaba mountain range. The areas evaluated in this group showed low floristic affinity with nearby areas, such as those evaluated near São Miguel Arcanjo, Capão Bonito and Sete Barras (Group 1), exhibiting the floristic heterogeneity of the region. The most frequent species in this group were $S$. terebinthifolius, Myrsine coriacea, Campomanesia guaviroba and M. splendens.

The fourth group comprised surveys conducted in seasonal semideciduous forests (near the cities of São Carlos, Campinas and Gália) and in a seasonal deciduous forest (near the city of Piracicaba), all within the state of São Paulo. These areas showed low floristic similarity with semideci- duous forests further south; hence, which appeared to have no significant influence on the vegetation at the Itaberá Ecological Station. The indicator species in this group were A. polyneuron, Myroxylon peruiferum, Ceiba speciosa and Metrodorea nigra.

The present study fell into the first group, the area evaluated showing low floristic affinity with stretches of the Araucaria forest near Campos do Jordão and in southern Brazil. The vegetation at the Itaberá Ecological Station showed greatest similarity to a stretch of seasonal semideciduous forest near the municipality of São Roque, in the state of São Paulo (Leite \& Rodrigues 2008), despite greater distance (approximately $210 \mathrm{~km}$ in a straight line) in comparison with the stretch of mixed rain forest near Barra do Chapéu and with the seasonal semideciduous forest near the municipality of Itatinga (approximately $100 \mathrm{~km}$ away in a straight line, located within the Paranapanema area, the same geomorphological unit as the Itaberá Ecological Station). These four surveys were grouped to form a subgroup, albeit showing low similarity among them, distinguished from other surveys of dense rain forest in São Paulo. The surrounding forest formations apparently had the greatest influence on areas of mixed rain forest in the southern part of the state of São Paulo, given that they proved to be isolated from other stretches of this formation, defining them as ecotones. The classification of the vegetation at the Itaberá Ecological Station as mixed rain forest is maintained for its physiognomic aspect (occurrence of A. angustifolia) rather than the floristic composition itself. This appears to be regional in scope, as was also observed by Souza (2008) in the Barra do Chapéu survey.

\section{Distribution of the species sampled at the Itaberá Ecological Station}

Of the 178 species sampled in our floristic and phytosociological surveys, 134 (74.9\%) were found in dense rain forest (26 species being exclusive to this formation); 129 (72.1\%) were found in seasonal semideciduous forest (16 being exclusive); 57 (31.8\%) were found in mixed rain forest (four being exclusive); 21 (11.7\%) were found in seasonal deciduous forest (one, Chomelia obtuse, being exclusive); $31(17.3 \%)$ were found in coastal woodland (restinga, none being exclusive); 19 (10.6\%) were found in savanna forest (cerradão) and cerrado stricto sensu (none being exclusive); and one (0.6\%), Trichilia pallida, was found to have a wide distribution in the Amazon, cerrado and Atlantic forest. There were 15 species that remained unclassified because of a lack of data in the literature, as well as their incomplete identification (Stehmann et al. 2009).

This floristic complexity, which translated to a mosaic of species of different origins, was expected, because, as previously mentioned, the Itaberá Ecological Station is within a region of ecological tension and is at an advanced successional stage, which, according to Klein (1960) favors 


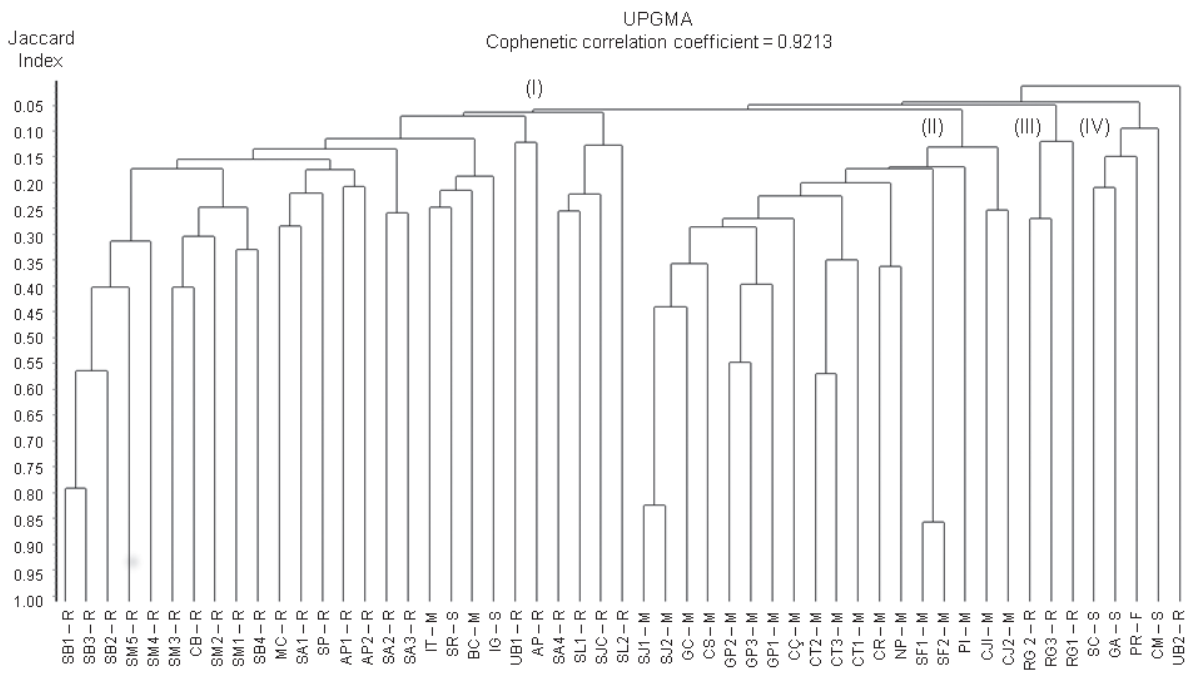

Figure 2. Dendrogram of floristic similarity between phytosociological surveys conducted in areas of dense rain forest (R), mixed rain forest (M), semideciduous forest (S) and deciduous forest (F) in southern Brazil and in the state of São Paulo, using the Jaccard index as the coefficient and the unweighted pair group method with arithmetic mean (UPGMA) to group the areas.

the invasion of broadleaf species from the highlands and forests of the Atlantic coast. However, it is again of note that the literature reviewed might contain errors, given the many controversies on the geographic distribution of these species among other literary sources and experts of recognized competence.

On the basis of the large number of species that were exclusive to dense rain forest and seasonal semideciduous forest, as well as the broader distribution of their exclusive species within the community, makes it obvious that there has been considerable invasion of the study area, in which typical mixed rain forest species have been supplanted by the invading species, altering the character of the area, i.e., making it less typical of a mixed rain forest. This pattern supports the hypothesis proposed by Klein (1960), in which the dynamic imbalance typical of mixed forests, in the current climate regime, would be caused by the greater competitive ability of the broadleaf forest species in the surrounding areas.

The process of forest fragmentation can reduce and isolate areas favorable to the survival of populations, resulting in local extinctions. In ecotones, that process can drastically alter the dynamics of plant communities and reduce regional biodiversity. The absence of characteristic elements of mixed rain forest in the lower stratum can influence the trajectory of areas subject to natural regeneration processes, such as forest clearing dynamics. In this scenario, open areas can be colonized by widely distributed pioneer species (generalist species common to the surrounding formations).

The impact of a reduction in area and of fragmentation can be mitigated by landscape management aimed at greater connectivity, enabling recolonization following local extinction, as well as facilitating the maintenance of metapopulations. Densification of species in danger of local extinction should also be considered as a means of avoiding genetic erosion of the remaining populations. For the mixed rain forest, even reforestation with A. angustifolia alone can be a viable alternative for greater connectivity, because the species acts as a catalyst for the regeneration of other species under its canopy.

If no management action is taken, the defining species of this formation will become locally extinct and the vegetation at the Itaberá Ecological Station will no longer be officially classified as mixed rain forest (Veloso 1991), but rather as a transitional community between dense rain forest and seasonal semideciduous forest. In other words, this fragment corresponds to what Klein (1960) called "mixed rain forest" or "mixed forest", consisting primarily of species characteristic of subtropical rain forest, with patches of pine occurring sporadically, mostly made up of older (adult or rachitic) individuals. Therefore, the current official classification of the vegetation at the Itaberá Ecological Station is based more on the few remaining $A$. angustifolia individuals than on the floristic composition itself, naturally tending to be abandoned for long periods or artificially sustained by management practices. The rigor of the official Brazilian classification of vegetation, as proposed by Veloso (1991), notwithstanding, a nomenclature more in line with the reality in the field would be "forest in transition from dense rain forest to seasonal semideciduous forest", given that $A$. angustifolia no longer dominates the phytophysiognomy of the unit.

\section{Acknowledgments}

This study received financial support in the form of research grants from the Brazilian Conselho Nacional de Desenvolvimento Científico e Tecnológico (CNPq, National Council 
for Scientific and Technological Development; "Mixed Rain Forest: assessment of plant diversity in Araucaria forests in the state of São Paulo" Project Grant no. 479084/2007). We thank the Itaberá Ecological Station employees for their help in the field. We are also grateful to the Forestry Institute for providing the necessary infrastructure, as well as to the biologist Rejane Esteves, as well as the taxonomists Geraldo Antônio Daher Correa Franco, Osny Tadeu Aguiar and João Batista Baitello, for their assistance.

\section{References}

$\mathrm{Ab}$ 'Saber, A.N. Os domínios de natureza no Brasil: potencialidades paisagísticas. Ateliê Editorial, São Paulo, SP. 2003.

Aguiar, O.T. Comparação entre métodos de quadrantes e parcelas na caracterização da composição florística e fitossociológica de um trecho de floresta ombrófila densa no Parque Estadual "Carlos Botelho" - São Miguel Arcanjo, São Paulo. 2003. Dissertação de Mestrado em Recursos Florestais. Escola Superior de Agricultura Luiz de Queiroz, Universidade de São Paulo, Piracicaba.

APG III. 2009. An update of the Angiosperm Phylogeny Group classification for the orders and families of flowering plants: APG III. Botanical Journal of the Linnean Society 161: 105-121.

Baitello. J.B.; Aguiar. O.T.; Rocha. F.T.; Pastore. J.A.; Esteves. R. 1992. Florística e fitossociologia do estrato arbóreo de um trecho da Serra da Cantareira (Núcleo Pinheirinho) - SP. Revista do Instituto Florestal 4: 291-298.

Cielo Filho, R.; Santin, D.A. 2002. Estudo florístico e fitossociológico de um fragmento florestal urbano, Bosque dos Alemães, Campinas, SP. Revista Brasileira de Botânica 25(3): 291-301.

Cientec. 2004. Mata Nativa: Sistema para análise fitossociológica e elaboração de planos de manejo de florestas nativas. Viçosa, Manual do usuário.

Cordeiro, J.; Rodrigues, W.A. 2007. Caracterização fitossociológica de um remanescente de Floresta Ombrófila mista em Guarapuava, PR. Revista Árvore 31: 545-554.

Custodio-Filho, A. 2002. Floresta Ombrófila Densa em diferentes altitudes no Parque Estadual Carlos Botelho, São Paulo, Brasil. Tese de Doutorado em Ecologia. Instituto de Biociências, Universidade de São Paulo, São Paulo.

Disa, A.C. 1993. Estrutura e diversidade do componente arbóreo e a regeneração do palmito (Euterpe edulis) em um trecho de mata secundária, no Parque Estadual de Carlos Botelho, SP. 1993, 126p. Dissertação de Mestrado em Recursos Florestais. Escola Superior de Agricultura "Luiz de Queiroz", Universidade de São Paulo, Piracicaba.

Dias, A.C.; Custodio-Filho, A.; Franco, G.A.D.C. 2000. Diversidade do componente arbóreo em um trecho de floresta secundária, Parque Estadual de Carlos Botelho, SP. Revista do Instituto Florestal 12( 2): $127-153$.

Dias, A.C. 2004. Composição florística, fitossociologia, diversidade de espécies arbóreas e comparação de métodos de amostragem na Floresta Ombrófila Densa do Parque Estadual de Carlos Botelho. SP, Brasil. 2004, 166p. Tese de Doutorado em Recursos Florestais. Escola Superior de Agricultura "Luiz de Queiroz", Universidade de São Paulo, Piracicaba.

Durigan, M.E. 1999. Florística, dinâmica e análise protéica de uma Floresta Ombrófila Mista em São João do Triunfo - PR. Curitiba. 1999, 125p. Dissertação de Mestrado em Ciência Florestal. Faculdade de Engenharia Florestal, Universidade Federal do Paraná, Curitiba.

Durigan, G.; Franco, G.A.D.C.; Saito, M.; Baitello, J.B. 2000. Estrutura e diversidade do componente arbóreo da floresta na Estação Ecológica de Caetetus, Gália, SP. Revista Brasileira de Botânica 23(4): 371-383.

Durigan, G. 2009. Estrutura e diversidade de comunidades florestais. Pp. 186-215. In: Martins, S.V. (Ed.). Ecologia de florestas tropicais do Brasil. Viçosa, Editora UFV.
Formento, S.; Schorn, L.A.; Ramos, A.B. 2004. Dinâmica estrutural de uma Floresta Ombrófila Mista em Campo Belo do Sul, SC. Revista Cerne 10( 2): 196-212.

Galetti, M. et al. 2010. The role of seed mass on the caching decision by agoutis, Dasyprocta leporina (Rodentia: Agoutidae). Zoologia 27: 472-476.

Godoy, J.R.L. 2001. Estrutura e composição específica da Mata Atlântica secundária de encosta sobre calcário e filito, no Parque Estadual Turístico do Alto Ribeira, Iporanga, SP. Dissertação de Mestrado em Ecologia. Instituto de Biociências, Universidade de São Paulo, São Paulo.

Gotelli, N.J. \& Colwell, R.K. 2001. Quantifying biodiversity:procedures and pitfalls in the measurement and comparison of species richness. Ecology Letters 4: 379-391.

Hosokawa, R.T. 1981. Manejo de florestas tropicais úmidas em regime de rendimento sustentado. UFPR. Curitiba. Relatório.

Instituto Brasileiro do Meio Ambiente e dos Recursos Naturais Renováveis. Listagem oficial das espécies vegetais brasileiras ameaçadas de extinção. Disponível em: < www.ibama.gov.br >. 1993. (Acesso em: 30/09/2010).

IUCN. IUCN Red List of Threatened Species. Version 2.3 (1994) and 3.1 (2001). Disponível em: < www.iucnredlist.org >. (Acesso em: 30/09/2010).

Ivanauskas, N.M.; Rodrigues, R.R.; Nave, A.G. 1999. Fitossociologia de um trecho de Floresta Estacional Semidecidual em Itatinga, São Paulo, Brasil. Scientia Florestalis 56: 83-99.

Ivanauskas, N.M.; Rodrigues, R.R. 2000. Florística e fitossociologia de remanescentes de floresta estacional decidual em Piracicaba, São Paulo, Brasil. Revista Brasileira de Botânica 23(3): 291-304,

Jardim Botânico do Rio de Janeiro. Lista de espécies da flora do Brasil. Ano 2010. Disponível em: <http://floradobrasil.jbrj.gov.br/2010>. Acesso entre Setembro de 2010 a Fevereiro de 2011.

Jarenkow, J.A.; Budke, J.C. 2009. Padrões florísticos e análise estrutural de remanescentes de florestas com araucária no Brasil. Pp.113-126. In: Fonseca. C.R.; Souza, A.F.; Leal-Zanchet, A.N.; Dutra, T.; Backes, A.; Ganado, G. (Eds.). Floresta com Araucária: ecologia, conservação e desenvolvimento sustentável. Ribeirão Preto, Editora Holos.

Klein, R.M. 1960. O aspecto dinâmico do pinheiro brasileiro. Sellowia 12: $17-44$.

Kozera, C.; Dittrich, V.A.O.; Silva, S.M. 2005a. Fitossociologia do componente arbóreo de um fragmento de Floresta Ombrófila Mista Montana, Curitiba, PR. Revista Floresta 36: 225-237.

Kozera, C.; Dittrich, V.A.O.; Silva, S.M. 2005b. Composição florística da floresta ombrófila mista montana do parque municipal do Barigüi, Curitiba, PR. Revista Floresta 36: 45-58.

Kronka, F.J.N.; Nalon, M.A.; Matsukuma, C.K.; Kanashiro, M.M.; Ywane, M.S.S.; Pavão, M.; Lima, L.M.P.R.; Guillaumon, J.R.; Baitello, J.B.; Barradas, A.M.F. 2005. Inventário Florestal da vegetação natural do Estado de São Paulo. Secretaria do Meio Ambiente: Instituto Florestal, Imprensa Oficial, São Paulo.

Leite, E.C.; Rodrigues, R.R. 2008. Fitossociologia e caracterização sucessional de um fragmento de floresta estacional no sudeste do Brasil. Revista Árvore 32(3): 583-595. Viçosa, MG.

Lima, R.A.F.; Moura, L.C. 2006. Canopy gap colonization in the Atlantic Montane Rain Forest. Brazilian Archives of Biology and Technology 49: 953-965.

Los, M.M. 2004. Florística, estrutura e diversidade de floresta com Araucária em áreas de diferentes tamanhos. Dissertação de Mestrado em Ecologia. Instituto de Biociências, Universidade de São Paulo, São Paulo.

Mantovani, W.; Rodrigues, R.R.; Rossi, L.; Romaniuc-Neto, S.; Catharino, E.L.M.; Cordeiro, I. 1991. A vegetação na serra do mar em Salesópolis. São Paulo, Academia de Ciências do Estado de São Paulo.

Nascimento, A.R.T.; Longhi, S.J.; Bena, D.A. 2001. Estrutura e padrões de distribuição espacial de espécies arbóreas em uma amostra de Floresta Ombrófila Mista em Nova Prata, RS. Ciência Florestal 11: 105-119.

Nascimento, F.H.F. 1994. A sucessão secundária inicial na Mata Atlântica, sobre a Serra de Paranapiacaba, Ribeirão Grande, SP. Dissertação de Mestrado em Ecologia. Instituto de Biociências, Universidade de São Paulo, São Paulo. 
Negrelle, R.A.B.; Silva, F.C. 1992. Fitossociologia de um trecho de floresta com Araucaria angustifolia (Bert.) O. Ktze. no município de CaçadorSC. Boletim de Pesquisa Florestal 24/25: 37-54.

Novais, P.S.; Rossi, M.; Mattos, I.F.A.; Kanashiro, M.M. 2009. Os solos da Estação Ecológica de Itaberá: Caracterização e mapeamento. IF Série Registros 40: 209-215.

Pielou, E.C. 1966. Introduction to mathematical ecology. New York, Wiley-Interscience.

Reginato, M.; Goldenberg, R. 2007. Análise florística, estrutural e fitogeográfica da vegetação em região de transição entre as Florestas Ombrófilas Mista e Densa Montana, Piraquara, Paraná, Brasil. Hoehnea 34: 349-364.

Rodrigues, R.R.; Ivanauskas, N.M.; Oliveira, A.A; Nogueira, A.; Castanho, C.T., Noreira, C.M.; Sampaio, D.; Franco, G.C; Batista, J.L.F.; Barreto, T.E.; Souza, V.C. 2004. Diversidade, dinâmica e conservação no Estado de São Paulo: 40ha de parcelas permanentes. Cananéia.

Rondon-Neto, R.M.; Kozera, C.; Andrade, R.R.; Cecy, A.T.; Hummes, A.P.; Fritzsons, E.; Caldeira, M.V.W.; Maciel, M.N.M.; Souza, M.K.F. 2002b. Caracterização florística e estrutural de um agrupamento de Floresta Ombrófila Mista em Curitiba, PR, Brasil. Revista Floresta 32: 3-16.

Rondon-Neto, R.M.; Watzlawick, L.F.; Caldeira, M.V.W.; Schoeninger, E.R. 2002a. Análise florística e estrutural de um fragmento de Floresta Ombrófila Mista Montana, Situada em Criúva, RS - Brasil. Ciência Florestal 12: 29-37.

Sanchez, M. 1999. Composição florística de um trecho de floresta ripária na Mata Atlântica em Picinguaba, Ubatuba, SP, Brasil. Revista Brasileira de Botânica 22 ( 1) 31-42.

Sanqueta, C.R.; Pizzato, W.; Péllico Neto, S.; Figueiredo Filho, A.; Eisfeld, R. L. 2002. Estrutura vertical de um fragmento de Floresta Ombrófila Mista no centro-sul do Paraná. Revista Floresta 32(2): 267-276.

SÃO PAULO. Resolução SMA 48 de setembro de 2004. Lista oficial das espécies da flora ameaçadas de extinção no Estado de São Paulo. Diário Oficial do Estado de São Paulo. Disponível em: <www.ibot.sp.gov. br>. (Acesso em: 30/09/2010).

Seger, C.D; Dlugosz, F.L.; Kurasz, G.; Martinez, D.T.; Ronconi, E.; Melo, L.A.N.; Bittencourt, S.M.; Brand, M.A.; Carniatto, I.; Galvão, F.; Roderjan, C.V. 2005. Levantamento florístico e análise fitossociológica de um remanescente de Floresta Ombrófila Mista localizado no município de Pinhais, Paraná, Brasil. Revista Floresta 35(2): 291-301.

Sentelhas, P.C.; Pereira, A.R.; Marin, F.R.; Angelocci, L.R.; Alfonsi, R.R. Caramori, P.H.; Swart, S. Balanços hídricos climatológicos do Brasil - 500: balanços hídricos de localidades brasileiras. Piracicaba, Escola Superior de Agricultura Luiz de Queiroz (ESALQ), 1999. CD- ROM.

Shepherd, G.J. 1995. FITOPAC 1. Manual do usuário. Campinas, Unicamp.

Silva, A.F. 1989. Composição florística e estrutura fitosociológica do estrato arbóreo da Reserva Florestal Prof. Augusto Ruschi, São José dos Campos-SP. Tese de Doutorado em Biologia Vegetal. Instituto de Biologia, Universidade Estadual de Campinas, Campinas. 1989.
Silva, D.W. 2003. Florística e Fitossociologia de dois remanescentes de Floresta Ombrófila Mista (Floresta com Araucária) e Análise de duas populações de Araucaria angustifolia (Bertol.) 0 . Kuntze na região de Guarapuava, PR. Tese de Doutorado em Ecologia. Instituto de Biociências, Universidade Federal de São Carlos, São Carlos.

Silva, L.A.; Soares, J.J. 2002. Levantamento fitossociológico em um fragmento de floresta estacional semidecídua, no município de São Carlos, SP. Acta Botanica Brasilica 16(2): 205-216.

Sonego, R.C.; Backes, A.E.; Souza, A.F. 2007. Descrição da estrutura de uma Floresta Ombrófila Mista, RS, Brasil, utilizando estimadores não-paramétricos de riqueza e rarefação de amostras. Acta Botanica Brasilica 21: 943-955.

Souza, R.P.M. 2008. Estrutura da comunidade arbórea de trechos de florestas de Araucaria no estado de São Paulo, Brasil. Dissertação de Mestrado em Recursos Florestais. Escola Superior de Agricultura Luiz de Queiroz - ESALQ/USP. Piracicaba.

Stehmann, J.R.; Forzza, R.C.; Salino, A.; Sobral, M.; Costa, D.P.; Kamino, L.H.Y. 2009. Plantas da Floresta Atlântica. Rio de Janeiro, Instituto de Pesquisas Jardim Botânico do Rio de Janeiro.

Tabarelli, M.; Villani, J.P.; Mantovani, W. 1994. Estudo comparativo de dois trechos de floresta secundária no núcleo Santa Virgínia, Parque Estadual da Serra do Mar, SP. Revista do Instituto Florestal 6: 1-11.

Tomasulo, P.L.B.; Cordeiro, I. 2000. Composição florística do Parque Municipal da Serra do Itapety, Mogi das Cruzes, SP. Boletim do Instituto de Botânica 14: 139-161.

Veloso, H.P.; Rangel Filho, A.L.R.; Lima, J.C.A. 1991. Classificação da vegetação brasileira adaptada a um sistema universal. Rio de Janeiro, Fundação IBGE.

Wanderley, M.G.L.; Shepherd, G.J.; Giulietti, A.M.; Melhem, T.S (Coordenadores). Flora fanerogâmica do estado de São Paulo. v.2, Instituto de Botânica, São Paulo-SP, 2002.

Wanderley, M.G.L.; Shepherd, G.J.; Giulietti, A.M.; Melhem, T.S (Coordenadores). Flora fanerogâmica do estado de São Paulo. v.3, Instituto de Botânica, São Paulo-SP, 2003.

Wanderley, M.G.L.; Shepherd, G.J.; Giulietti, A.M.; Melhem, T.S (Coordenadores). Flora fanerogâmica do estado de São Paulo. v.4, Instituto de Botânica, São Paulo-SP. 2005.

Wanderley, M.G.L.; Shepherd, G.J.; Giulietti, A.M.; Melhem, T.S (Coordenadores). Flora fanerogâmica do estado de São Paulo. v.5, Instituto de Botânica, São Paulo-SP. 2007.

Wanderley, M.G.L.; Shepherd, G.J.; Giulietti, A.M.; Melhem, T.S (Coordenadores). Flora fanerogâmica do estado de São Paulo. v.6, Instituto de Botânica, São Paulo-SP. 2009.

Watzlawick, L.F.; Sanquetta, C.R.; Valerio, A.F.; Silvestre, R. 2005. Caracterização da composição florística e estrutura de uma Floresta Ombrófila Mista, no município de General Carneiro, PR. Ambiência 1(2): 229-238. 